\title{
Understanding Fetal Hemodynamics Using Cardiovascular Magnetic Resonance Imaging
}

\author{
Liqun Sun $^{\mathrm{a}}$ Davide Marini ${ }^{\mathrm{a}}$ Brahmdeep Saini $^{\mathrm{a}}$ Eric Schrauben ${ }^{\mathrm{c}}$ \\ Christopher K. Macgowan ${ }^{c, d}$ Mike Seed ${ }^{a, b}$ \\ a Division of Cardiology, Hospital for Sick Children, Toronto, ON, Canada; ${ }^{\mathrm{b}}$ Department of Paediatrics and Medical \\ Imaging, University of Toronto, Toronto, ON, Canada; ' Division of Translational Medicine, Hospital for Sick Children, \\ Toronto, ON, Canada; ${ }^{\mathrm{d}}$ Department of Medical Biophysics, University of Toronto, Toronto, ON, Canada
}

\section{Keywords}

Fetal hemodynamics $\cdot$ Cardiovascular MRI $\cdot$ MRI oximetry

\begin{abstract}
Human fetal circulatory physiology has been investigated extensively using grey-scale ultrasound, which provides excellent visualization of cardiac anatomy and function, while velocity profiles in the heart and vessels can be interrogated using Doppler. Measures of cerebral and placental vascular resistance, as well as indirect measures of intracardiac pressure obtained from the velocity waveform in the ductus venosus are routinely used to guide the management of fetal cardiovascular and placental disease. However, the characterization of some key elements of cardiovascular physiology such as vessel blood flow and the oxygen content of blood in the arteries and veins, as well as fetal oxygen delivery and consumption are not readily measured using ultrasound. To study these parameters, we have historically relied on data obtained using invasive measurements made in animal models, which are not equivalent to the human in every respect. Over recent years, a number of technical advances have been made that have allowed us to examine the human fetal circulatory system using cardiovascular magnetic resonance (CMR). The combination of vessel blood flow mea-
\end{abstract}

surements made using cine phase contrast magnetic resonance imaging and vessel blood oxygen saturation and hematocrit measurements made using T1 and T2 mapping have enabled us to emulate those classic fetal sheep experiments defining the distribution of blood flow and oxygen transport across the fetal circulation in the human fetus. In addition, we have applied these techniques to study the relationship between abnormal fetal cardiovascular physiology and fetal development in the setting of congenital heart disease and placental insufficiency. CMR has become an important diagnostic tool in the assessment of cardiovascular physiology in the setting of postnatal cardiovascular disease, and is now being applied to the fetus to enhance our understanding of normal and abnormal fetal circulatory physiology and its impact on fetal well-being.

(c) 2020 S. Karger AG, Basel

\section{Background}

Early investigations into the circulatory physiology of fetal sheep include those undertaken in the 1930s by Sir Joseph Barcroft at Cambridge University [1]. Sir Geoffrey Dawes elaborated on this work and conducted the first detailed studies of the fetal circulation [2]. Rudolph and karger@karger.com

www.karger.com/fdt

(C) 2020 S. Karger AG, Basel

Karger ${ }^{\prime}=$
Mike Seed, MD

Division of Cardiology, The Hospital for Sick Children

555 University Avenue

Toronto, ON M5G 1X8 (Canada)

mike.seed@sickkids.ca 
Heymann adopted the techniques pioneered by Barcroft and Dawes for exteriorizing and catheterizing fetal sheep and developed a method for making accurate measurements of the distribution of blood flow in fetal lambs using radioactively labelled microspheres [3]. Their measures of fetal blood pressure, arterial and venous blood gases, absolute blood flow, and proportions of the combined ventricular output (CVO) delivered to the pulmonary, cerebral, and placental circulations, as well as measurements of the magnitude of the shunts across the foramen ovale, ductus arteriosus, and ductus venosus have provided us with our modern understanding of the normal fetal circulation [4]. The mammalian fetal circulation operates in parallel, not series, with shunts at ductus venosus, foramen ovale, and ductus arteriosus which allow blood returning from the umbilical vein to bypass the liver and lungs. The presence of these unique shunts results in the possibility for the right and left ventricles to have different cardiac outputs. In sheep, the right ventricle provides the more dominant contribution towards the combined ventricular output (CVO). By contrast with the postnatal circulation, the presence of the ductus arteriosus also results in equalization of blood pressure on both sides of the heart. Arterial oxygen saturations are considerably lower in the fetus than postnatally, with the highest oxygen saturations of approximately $85 \%$ found in the umbilical vein. The preferential flow, or streaming, of oxygenated blood from the umbilical vein and ductus venosus towards the foramen ovale results in higher saturations in the left heart compared with the right. Hypoxemic blood delivered to the right heart is directed back to the placenta via the ductus arteriosus, descending aorta, and umbilical arteries, while the relatively low oxygen content of the blood supplied to the fetal lungs is largely responsible for the vasoconstricted state of the pulmonary circulation.

Fetal sheep studies have also been used to investigate fetal circulatory adaptations to acute hypoxia, whereby the presence of fetal shunts and differential changes in the cerebral, coronary, pulmonary, and systemic circulations result in redistribution of blood flow to favor conservation of myocardial and cerebral oxygen delivery at the expense of the musculoskeletal system, abdominal viscera, and lungs [5]. This response, which is detectable in humans using ultrasound through changes in cerebral arterial pulsatility, is frequently referred to as "brain-sparing physiology" [6]. However, in the setting of chronic hypoxemia, there is some return to more normal flow distribution (and cerebral arterial Doppler velocity profiles) with secondary adaptations to reduced oxygen delivery

Fetal Cardiovascular Magnetic Resonance Imaging including the slowing of growth and reductions in fetal activity [7]. These secondary adaptations are associated with reductions in fetal oxygen consumption, which presumably serve to protect the fetus against hypoxic injury.

Over recent decades, magnetic resonance imaging (MRI) techniques for assessing cardiovascular function and hemodynamics in postnatal patients have played an increasingly important role in clinical practice [8]. Anatomical cine cardiovascular magnetic resonance (CMR) provides the most accurate means to measure ventricular volumes and ejection fraction, while cine phase contrast flow assessment represents the noninvasive gold standard for the measurement of vessel flow, providing unique quantification of valvular regurgitation and shunt volume [9]. In conjunction with invasive pressure measurements, this approach allows for the precise measurement of systemic and pulmonary vascular resistance [10].

CMR provides approaches to myocardial tissue characterization, including the quantification of regional and diffuse myocardial fibrosis as well as techniques for measuring myocardial perfusion. The opposing magnetic properties of oxy- and deoxy-hemoglobin can be exploited for direct visualization and quantification of tissue and blood oxygenation [11]. One approach to magnetic resonance oximetry based on blood relaxometry involves the application of a T2 preparation sequence for the quantification of T2 combined with a modified Look-Locker inversion recovery (MOLLI) sequence for measuring the T1 of blood [12]. The combination of any pair of vessel $\mathrm{T} 1$ and $\mathrm{T} 2$ measurements provides an accurate measurement of oxygen saturation and hematocrit in vitro, although in vivo validation of this approach to fetal oximetry has not yet been provided. The measurement of the T2 of flowing blood is challenging, and requires careful attention to the refocusing interval, choice of T2 preparation times, and consideration of artifacts arising from turbulent flow, local variations in the magnetic field, and partial voluming. However, assuming these challenges can be overcome and ignoring the negligible contribution of dissolved oxygen to the oxygen content of fetal blood, oxygen content can be quantified as the product of oxygen saturation and hematocrit by 1.36 (the amount of oxygen in milliliters bound to $1 \mathrm{~g}$ of hemoglobin). Fetal oxygen delivery can then be calculated as the product of umbilical vein oxygen content and umbilical blood flow, while fetal oxygen consumption is the product of umbilical venous flow by the umbilical venoarterial difference in oxygen content.

The adaptation of CMR techniques for use in fetal subjects has required technical development to counter arti- 
facts arising from the high heart rates, small size, and a variety of maternal and fetal movements that degrade image quality. Unlike postnatal cardiac MRI, which utilizes the electrocardiographic (ECG) signal to synchronize the collection of data across the cardiac cycle, fetal CMR requires alternative approaches to ECG gating. Recently, a combination of metric-optimized gating, motion correction algorithms, and accelerated imaging acquisitions has shown promise in overcoming these challenges, resulting in high-resolution fetal cardiac imaging $[13,14]$. This kind of approach has been particularly effective for imaging the late-gestation fetus, which is larger and relatively restricted in terms of fetal motion, while the inherent trade-off between achieving adequate signal-to-noise ratio and spatial resolution while limiting artifact arising from more vigorous fetal motion remains a challenge in early pregnancy [12-23].

MRI appears to be safe for use in pregnancy, although gadolinium contrast agents should be avoided due to the increased risk of rheumatological, inflammatory, or infiltrative skin conditions, stillbirth, and neonatal death, and the American College of Radiology guidelines recommend that MRI should be limited to those situations when it offers additional information over ultrasound $[24,25]$.

\section{Human Fetal Circulatory Physiology by MRI}

\section{Normal Fetal Circulation}

Using a combination of blood flow measurements made with cine phase contrast MRI with metric optimized gating and oxygen saturation measurements made using T2 mapping, we have collected preliminary reference ranges for the distribution of blood flow and oxygen transport in the late-gestation human fetus [26]. The results are in keeping with previous estimates regarding the human fetal circulation made by Rudolph which were based on his invasive measurements made in fetal sheep [4]. Pulmonary and cerebral blood flow are higher in the human fetus compared with the sheep fetus, the latter being readily explained by the larger size of the human brain compared with the sheep. Umbilical flow is lower in the human, although fetal oxygen delivery is similar due to the higher fetal hematocrit levels in the human [4] (Fig. 1).

In common with the sheep fetus, the oxygen saturations of the blood in the late-gestation human left heart and ascending aorta (65\%) are approximately $15 \%$ higher than in the right ventricle and pulmonary artery (50\%). Human fetal MRI oximetry data therefore support the presence of the same streaming of oxygenated blood from the umbilical vein to the left heart via the ductus venosus and foramen ovale that we have demonstrated in fetal sheep using 4D flow MRI (a volumetric cine phase contrast MRI technique) [27]. The hemodynamics of the ductus venosus result in a remarkable mechanism whereby two columns of blood moving at different speeds and with different oxygen saturations coexist in the suprahepatic inferior vena cava. The well-oxygenated blood returning from the placenta via the ductus venosus is directed toward the foramen ovale, presumably to ensure an adequate supply of glucose and oxygen to the brain and heart, the most metabolically active organs in utero. Streaming also results in transport of more deoxygenated blood back to the placenta via the ductus arteriosus, descending aorta, and umbilical arteries. The deoxygenated blood that passes into the right heart and pulmonary trunk is also presented to the pulmonary circulation, where its relatively low oxygen content maintains the high pulmonary vascular resistance typical of the fetal circulation [4] (Fig. 2).

\section{Fetal Congenital Heart Disease}

Using a combination of MRI blood flow and oximetry measurements, concepts regarding the impact of cardiac malformations and placental dysfunction on fetal circulatory physiology have been examined. Mean vessel flows and oxygen saturations obtained using the MRI techniques described above in a preliminary group of fetuses with a range of more severe forms of congenital heart disease (CHD) reveal many of the expected changes in fetal circulatory physiology [26]. For example, when one side of the heart is obstructed, there is a compensatory increase in blood flow through the unobstructed side. For example, in hypoplastic left heart syndrome, ascending aortic flow is significantly reduced, while flow across the main pulmonary artery and ductus arteriosus is markedly increased. Similarly, in lesions characterized by right heart obstruction like tetralogy of Fallot and tricuspid atresia, we have observed significant increases in ascending aortic flow with diminished flow in the main pulmonary artery and arterial duct. In transposition of the great arteries we have noted a reversal of the normal relationship between main pulmonary artery and ascending aortic flows. In the normal fetal circulation, main pulmonary artery flow exceeds ascending aortic flow. This is possible in the fetal circulation because of the presence of shunts at the foramen ovale and ductus arteriosus, and presumably reflects the greater venous return to the right ventricle. In transposition, ascending aortic flow is higher 


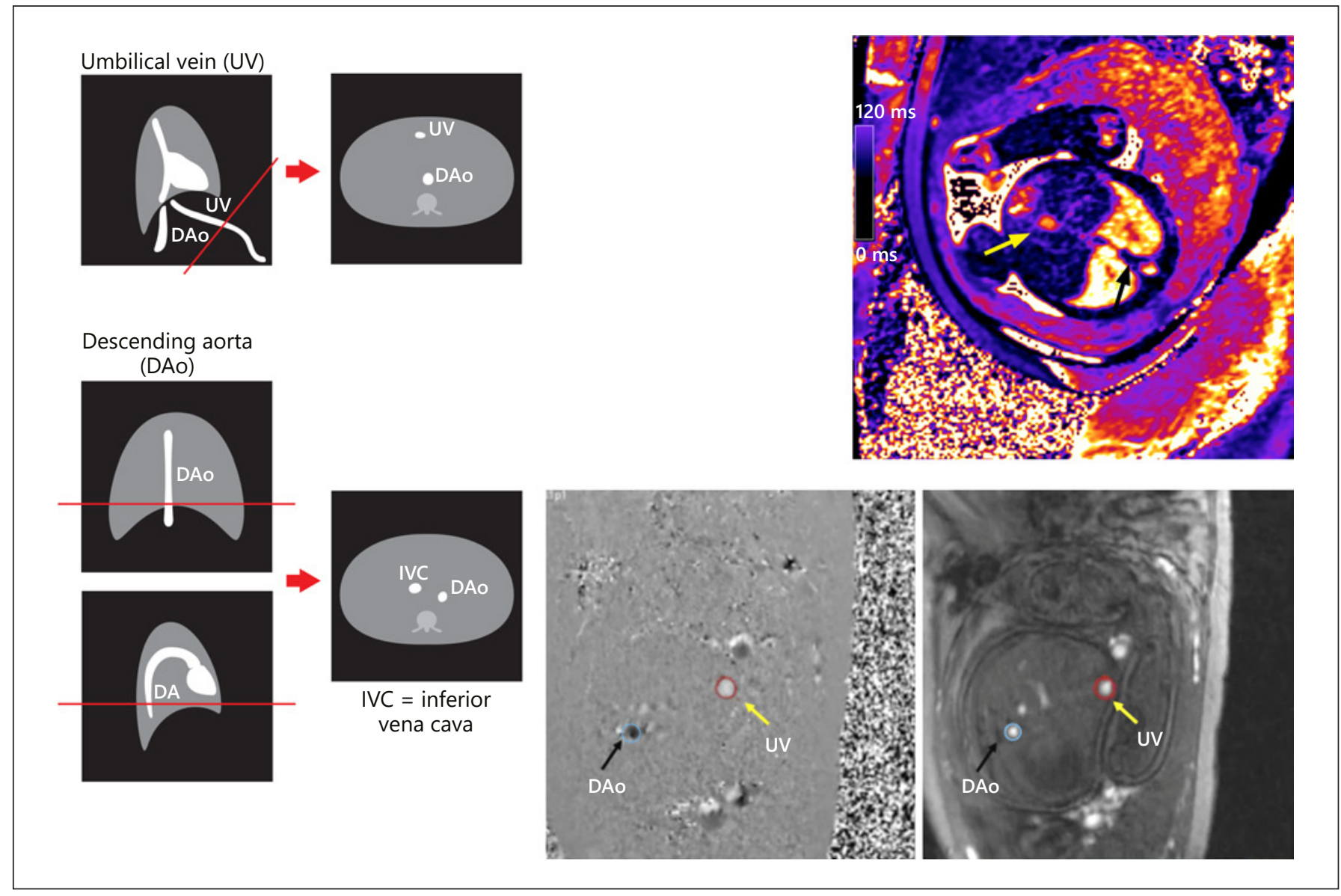

Fig. 1. Oxygen saturation and blood flow in the umbilical vein and descending aorta of a normal late-gestation human fetus by T2 mapping (top right) and cine phase contrast magnetic resonance imaging (bottom). The short-axis imaging planes for umbilical vein and descending aorta flow quantification and oximetry are pre-

than main pulmonary artery flow, likely due to the discordant ventriculoarterial connections that result in the aorta being connected to the dominant right ventricle. Despite these variations in the outputs of the right and left heart, fetal organ perfusion appears to be reasonably well maintained. For example, pulmonary blood flow is rather stable across all of these forms of $\mathrm{CHD}$, and similar to pulmonary blood flow in normal controls. If superior vena caval flow is taken as a surrogate for cerebral blood flow, then cerebral blood flow is also well maintained in most forms of CHD. The exception to this may be in those fetuses with more severe forms of Ebstein's anomaly. Fetal Ebstein's anomaly is associated with the lowest CVOs we have observed in fetuses with $\mathrm{CHD}$, and appears to be prone to lower cerebral blood flow. Other types of CHD with single ventricle physiology are also associated with a scribed from orthogonal long-axis views of the vessels (top left). The higher signal in the umbilical vein in the $\mathrm{T} 2 \mathrm{map}(\mathrm{T} 2=200 \mathrm{~ms}$, $\mathrm{SaO}_{2}=80 \%$ ) indicates the higher oxygen saturation of the blood returning from the placenta compared with the blood returning to the placenta via the descending aorta $\left(\mathrm{T} 2=104 \mathrm{~ms}, \mathrm{SaO}_{2}=51 \%\right)$.

reduction in CVO in the range of $10-20 \%$. While cerebral and pulmonary perfusion is relatively well maintained, this reduction in cardiac output is associated with a drop in umbilical blood flow.

We hypothesized that the changes in fetal brain metabolism and reduced newborn brain size associated with $\mathrm{CHD}$, as demonstrated by Limperopoulos et al. [28], result from reductions in cerebral oxygenation arising from interruption of the normal streaming of oxygenated blood from the placenta to the fetal brain. Our findings confirmed an average $10 \%$ reduction in the oxygen saturation of blood supplied to the developing brain in subjects with complex CHD [29]. The mechanisms leading to this aortic desaturation depend on the cardiac anatomy. For example, in fetuses with transposition of the great arteries, we found that the normal streaming of blood 


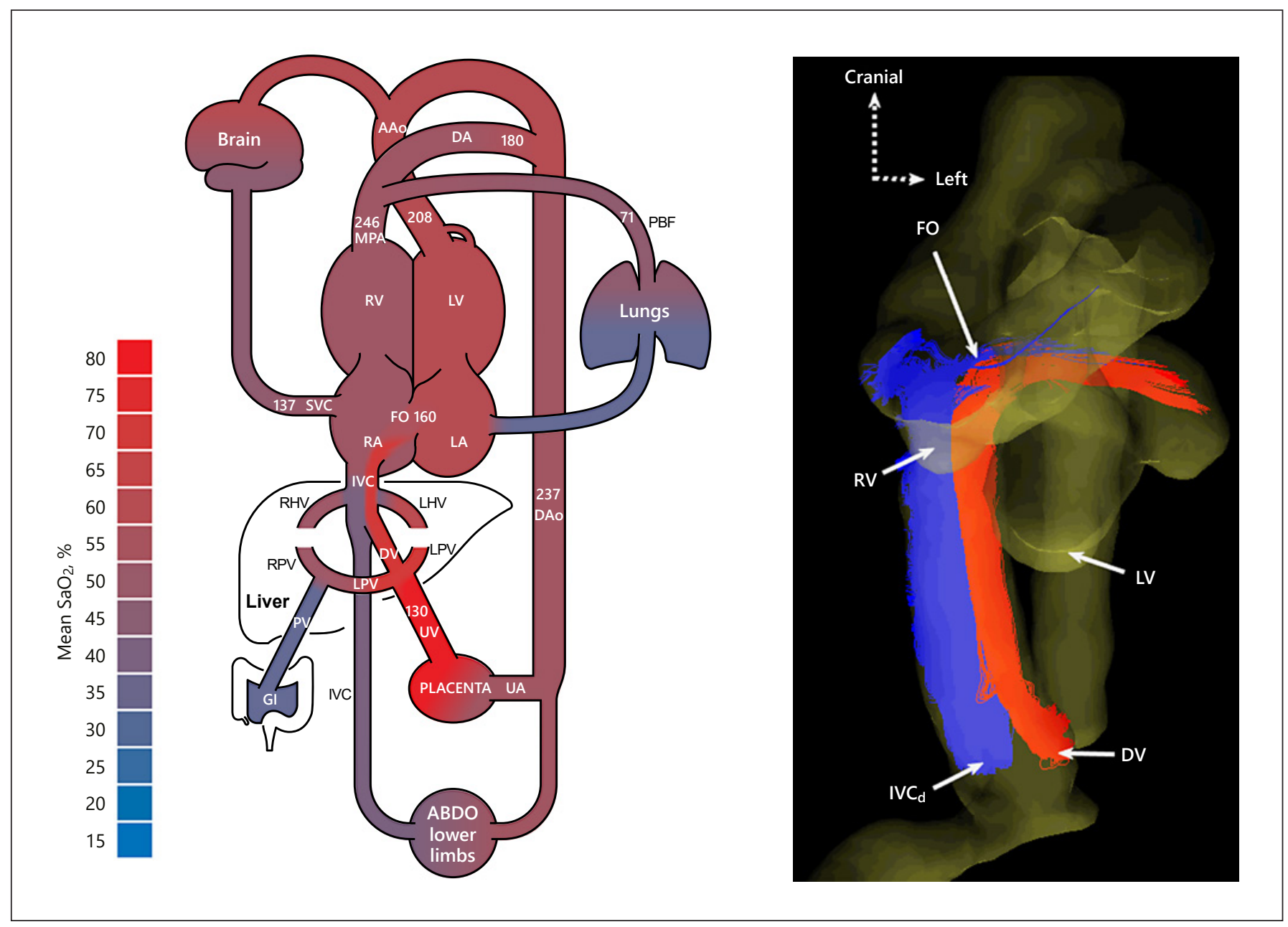

Fig. 2. Oxygen transport in the human (left) and sheep fetus (right). Blood flows $(\mathrm{mL} / \mathrm{min} / \mathrm{kg}$ ) are indicated by the numbers in the vessels, while oxygen saturations are indicated by the color coding, confirming higher oxygen saturations in the left heart than the right (left). The streaming of well-oxygenated blood from the ductus venosus (DV) across the foramen ovale (FO) seen on this fetal sheep 4D flow acquisition is responsible for higher oxygen saturations in the left heart compared with the right. UV, umbilical vein;
IVCd, distal inferior vena cava; SVC, superior vena cava; RA, right atrium; LA, left atrium; RV, right ventricle; LV, left ventricle; MPA, main pulmonary artery; DA, ductus arteriosus; AAo, ascending aorta; DAo, descending aorta; RHV, right hepatic vein; LHV, left hepatic vein; PV, portal vein; RPV, right portal vein; LPV, left portal vein; PBF, pulmonary blood flow. Figure modified from Schrauben et al. [27] used under CC BY. from the ductus venosus to the left atrium results in welloxygenated blood being directed to the pulmonary circulation, while the blood supplied to the brain is derived largely from more deoxygenated blood returning from the caval veins (Fig. 3). In fetuses with tetralogy of Fallot, the oxygenated blood crossing the foramen ovale passes into the left ventricle and aorta in the normal way but is diluted by more deoxygenated blood shunting from right to left across the ventricular septal defect. In hypoplastic left heart syndrome, no streaming of oxygenated blood is possible because there is essentially only one outlet from the heart, so the entire fetal circulation is supplied by blood with the same oxygen content. In addition to the disruption of streaming of oxygenated blood toward the brain, in single-ventricle hearts oxygen delivery to the fetus is diminished through a reduction in CVO. For example, in fetuses with more severe forms of Ebstein's anomaly, fetal CMR reveals a reduction in CVO of up to $50 \%$ compared with normal fetuses [30, 31]. While Ebstein's anomaly subjects appear to have reasonably normal placental function, their lower CVO results in reduced umbilical flow which results in reduced fetal oxy- 
Fig. 3. Streaming in fetal transposition. The higher T2 signal in the left ventricle (LV) and main pulmonary artery $(\mathrm{T} 2=117 \mathrm{~ms}$, $\mathrm{SaO}_{2}=57 \%$ ) compared with the lower signal in the right ventricle (RV) and aorta (T2 $=95 \mathrm{~ms}, \mathrm{SaO}_{2}=50 \%$ ) indicates that welloxygenated blood is being directed away from the brain and towards the lungs. MPA, main pulmonary artery; AAo, ascending aorta.

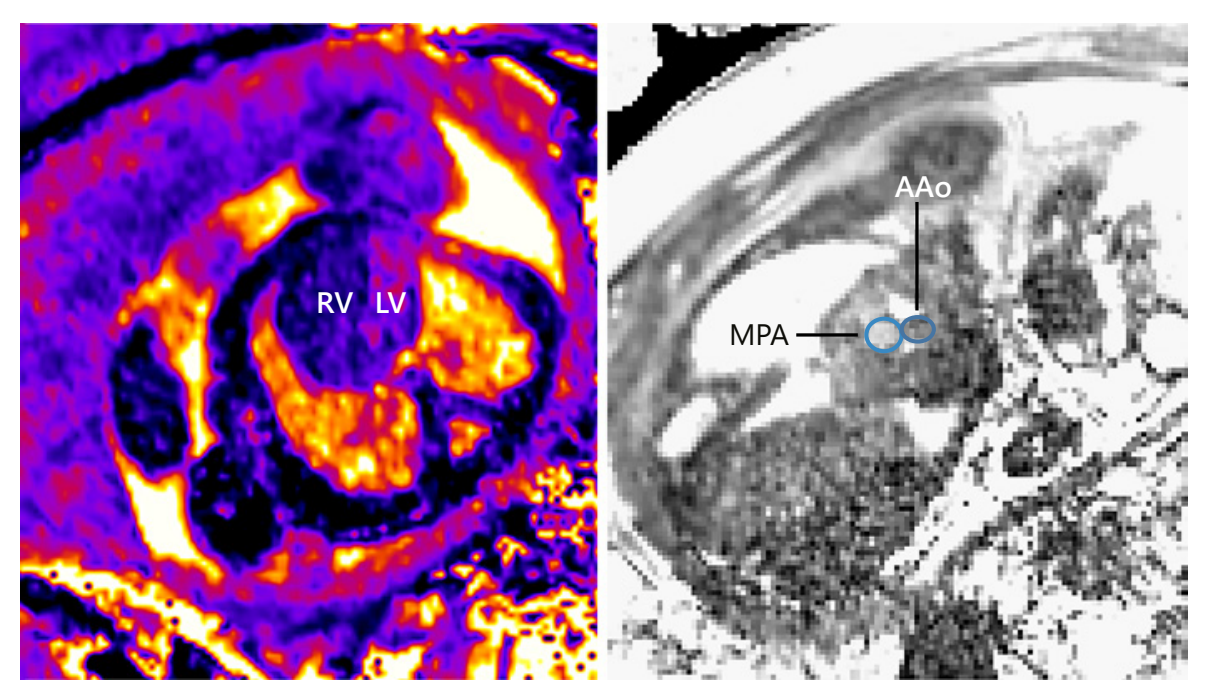

gen delivery. Although fetuses with Ebstein's anomaly demonstrate increases in oxygen extraction, which partially compensates for the reduced oxygen delivery, body and brain growth remains impaired. In addition to the reductions in umbilical flow seen in fetuses with singleventricle hearts, CHD appears to be associated with modest reductions in the oxygen content of umbilical venous blood. This may reflect abnormal structural differences that have been described on pathologic examination of the placenta in pregnancies affected by fetal CHD [3234]. It is interesting to consider whether underdevelopment of the fetal placental vasculature resulting from impaired fetal perfusion of the placenta may result in a unique form of placental insufficiency.

Our fetal CMR findings in patients with CHD appear to support the concept that the disordered prenatal brain growth that is typical of CHD could be caused by the disruption of normal fetal cardiovascular physiology resulting in diminished substrate delivery. Recent fetal brain blood oxygen level-dependent (BOLD) MRI data are also in keeping with reductions in cerebral tissue oxygenation in fetuses with CHD [35]. However, recent deep genetic analyses have revealed widespread DNA damage in patients with CHD, which is more severe in the setting of associated noncardiac organ system malformations and neurodevelopmental delay [36]. Interestingly, some of these de novo mutations are also found in children with neurodevelopmental problems having normal hearts. These results have led to speculation that the neurodevelopmental problems commonly affecting nonsyndromic children with $\mathrm{CHD}$ are more likely to be genetic in origin rather than the result of abnormal fetal physiology or

Fetal Cardiovascular Magnetic Resonance Imaging postnatal hemodynamics and brain injury. One approach to testing this hypothesis is to attempt to augment fetal brain oxygenation using maternal hyperoxygenation in fetuses with CHD. Using T2 mapping, we observed significant increases in the oxygen content of human umbilical venous blood during an episode of acute maternal hyperoxygenation, raising the possibility that cerebral oxygenation could be improved in fetuses with some forms of $\mathrm{CHD}$ through inhaled maternal supplemental oxygen [37]. A trial investigating the potential neuroprotective efficacy of chronic maternal hyperoxygenation in fetuses with single-ventricle $\mathrm{CHD}$ is being conducted at our center, although one study of chronic intermittent maternal hyperoxygenation in the setting of left ventricular hypoplasia found a reduction in brain size in fetuses treated with oxygen [38].

\section{Fetal Growth Restriction}

CMR has been used to study placental and fetal cardiovascular physiology in intrauterine growth restriction (IUGR) [39]. In fetuses with late-onset IUGR, umbilical vein oxygen saturation and flow are reduced, resulting in diminished fetal oxygen delivery, which is associated with lower fetal oxygen consumption. IUGR fetuses have lower oxygen saturations in the ascending aorta and higher superior vena caval flow, in keeping with fetal animal models of "brain-sparing physiology" (Fig. 4). By contrast, increased oxygen extraction and a more modest reduction in oxygen saturations in the main pulmonary artery are associated with reductions in pulmonary blood flow, in keeping with hypoxic pulmonary vasoconstriction. Although the correlation we observed between umbilical 


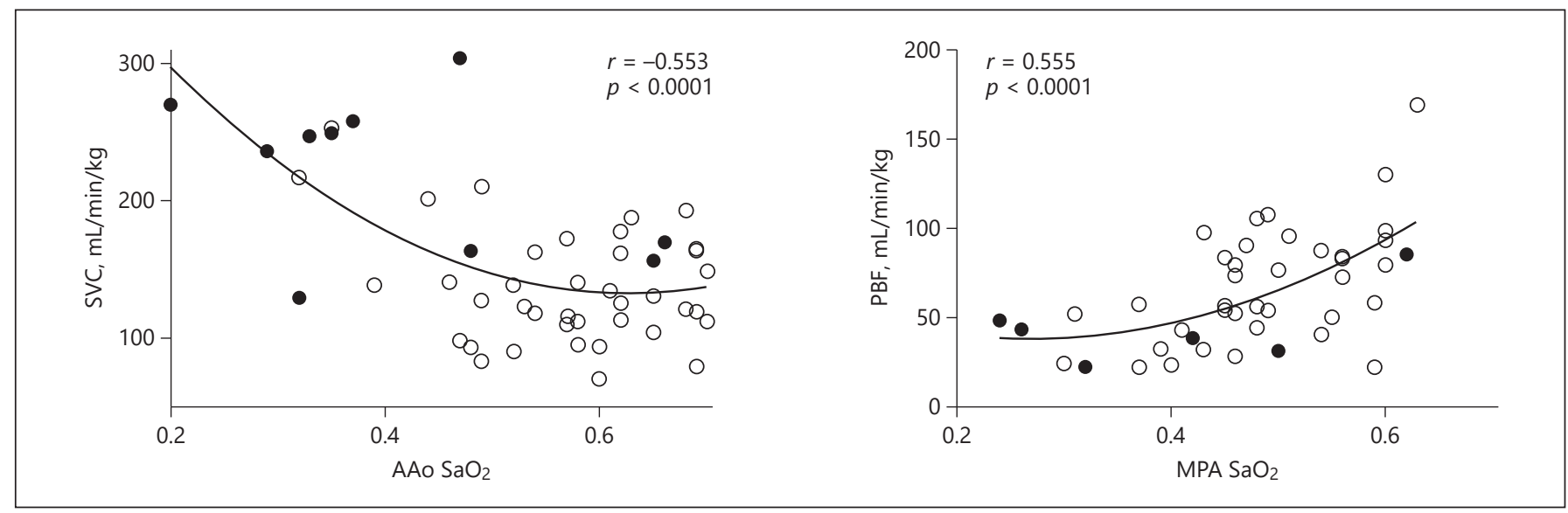

Fig. 4. Relationships between vessel oxygen saturation and flow by $\mathrm{T} 2$ mapping and phase contrast magnetic resonance imaging in a cohort of 50 late-gestation human fetuses - 10 IUGR fetuses (IUGR group, mean \pm SD gestational age $35.7 \pm 1.7$ weeks (solid circles) and 40 control fetuses $(37.0 \pm 1.1$ weeks; open circles). In

vein oxygen saturation and flow is less striking, it is perhaps in keeping with the notion that placental disease is associated with stealing of diastolic blood flow into the cerebral circulation, high placental resistance, reduced umbilical flow, and impaired placental oxygen exchange.

Despite showing evidence of brain-sparing, newborns with IUGR have smaller brains than controls, in keeping with the brains of newborns with CHD. The relative importance of these delays in brain growth and maturation compared with postnatal physiologic and environmental factors has yet to be determined. However, the interpretation of studies investigating the relationship between placental insufficiency and neurodevelopmental outcomes has often been complicated by difficulties in distinguishing between milder forms of placental insufficiency and constitutional small size for gestational age. Of interest, in our study of late-onset IUGR, which used a composite scoring system incorporating fetal growth and Doppler parameters, placental histology, and neonatal nutrition to diagnose the presence of fetal growth restriction, $1 / 3$ of the fetuses with late-onset IUGR had normal blood flow distribution by MRI and normal cerebroplacental ratio by Doppler. We believe that this group of fetuses, which likely have a very stable and milder form of placental insufficiency, may frequently go undetected in clinical practice.

The improved sensitivity of fetal MRI to chronic fetal hypoxia and its versatility for looking at placental, circulatory, and cerebral physiology and brain development may be helpful as new approaches to improving the outcomes of fetuses exposed to a hostile in utero environ- fetuses with lower oxygen saturations, there is redistribution of the fetal circulation with increased cerebral blood flow and reduced pulmonary flow. SVC, superior vena cava; PBF, pulmonary blood flow; AAo, ascending aorta; MPA, main pulmonary artery.

ment are sought. A number of MRI methods for investigating placental physiology in human and animal models are currently being explored, with the broad aim of addressing the relatively poor in vivo characterization of placental pathology and limited options for treating placental disease that are currently available. Dynamic contrast-enhanced MRI has been used to quantify placental perfusion in animal models [40]. Other techniques including placental BOLD and magnetic resonance spectroscopy have also been used to explore placental function $[41,42]$.

\section{Future Developments}

The development of fetal CMR has provided a new research tool for studying fetal cardiovascular physiology and the impact of abnormal circulatory hemodynamics on fetal development [30, 32, 43-46]. Some centers have suggested there may be a clinical role for fetal CMR in the diagnosis of congenital malformations affecting the arterial and venous anatomy $[22,23]$. Potential future clinical indications for fetal CMR also include improved diagnosis of fetal cardiac malformations, the monitoring of fetal therapy, and the assessment of placental function. Lloyd et al. [47] have explored the potential role of fetal CMR for the evaluation of complex cardiac anatomy, particularly in late gestation, when ultrasound imaging of the fetal heart may be hampered by adverse fetal lie, oligohydramnios, and reflection of the ultrasound beam by the 
bony thorax. As the range of fetal treatments available broadens, the need for more advanced fetal cardiovascular imaging may emerge as a means of therapeutic monitoring. For example, cine phase contrast flow quantification has been used to demonstrate an increase in pulmonary blood flow following decompression of pulmonary venous obstruction in fetuses with hypoplastic left heart syndrome with restrictive atrial septum [45]. Similarly, improvements in fetal cardiac output have been demonstrated following transplacental nonsteroidal anti-inflammatory drug-induced ductal constriction in fetuses with Ebstein's anomaly complicated by a circular shunt [31]. MRI has also been used to interrogate the potential to enhance the growth of underdeveloped left heart structures through the pulmonary vasodilatory action of maternal hyperoxygenation [46]. Finally, preliminary work on the development of MRI measurement of fetal hematocrit indicates that in future, decision-making about the need for repeat blood transfusions in the setting of fetal anemia could be guided by MRI $[48,49]$.

Fetal CMR techniques developed for human imaging may also be useful for studying animal models of human disease and for evaluating experimental fetal therapies in animal models. Very high quality measurements of blood flow and blood oxygen content are possible using a blood pressure waveform to trigger the MRI acquisition. The pressure trace is obtained using an indwelling fetal arterial catheter and imaging quality is optimized using maternal anesthesia to restrict maternal and fetal motion. Examples of applications of fetal CMR that have been used to study animal models include the visualization of myocardial infarcts induced by coronary artery ligation and the quantification of the distribution of blood flow across the fetal sheep circulation $[50,51]$. Thus, the fetal sheep physiology work that inspired the development of human fetal CMR may now be enhanced by this versatile imaging medium for examining cardiovascular physiology, which now provides an alternative to conventional invasive techniques.

\section{Statement of Ethics}

The research ethics board of the Hospital for Sick Children approved the study, and written consent was obtained from every mother prior to study enrolment.

\section{Disclosure Statement}

The authors declare that they have no conflicts of interest.

\section{Funding Sources}

None.

\section{Author Contributions}

Manuscript writing and editing: L.S., D.M., B.S., E.S., C.K.M., and M.S.; MRI data analysis: L.S. and B.S.; fetal MRI scanning: D.M. and M.S.

\section{References}

1 Roughton FJW. Joseph Barcroft 1872-1947. R Soc Obstet Notices. 1948;6:315-45.

2 Dawes G. The physiological control of the fetal circulation. Obstet Gynecol Surv. 1961 Feb;16(1):20-5.

3 Rudolph AM, Heymann MA. The circulation of the fetus in utero. Methods for studying distribution of blood flow, cardiac output and organ blood flow. Circ Res. 1967 Aug;21(2):163-84.

4 Rudolph A. Congenital Diseases of the Heart: Clinical-Physiological Considerations. 3rd ed. Chichester, UK: Wiley Blackwell; 2009. https://doi.org/10.1002/9781444311822.

5 Cohn HE, Sacks EJ, Heymann MA, Rudolph AM. Cardiovascular responses to hypoxemia and acidemia in fetal lambs. Am J Obstet Gynecol. 1974 Nov;120(6):817-24.

6 Rurak DW, Richardson BS, Patrick JE, Carmichael L, Homan J. Oxygen consumption in the fetal lamb during sustained hypoxemia with progressive acidemia. Am J Physiol. 1990 May;258(5 Pt 2):R1108-15.
7 Richardson BS, Bocking AD. Metabolic and circulatory adaptations to chronic hypoxia in the fetus. Comp Biochem Physiol A Mol Integr Physiol. 1998 Mar;119(3):717-23.

8 Fratz S, Chung T, Greil GF, Samyn MM, Taylor AM, Valsangiacomo Buechel ER, et al. Guidelines and protocols for cardiovascular magnetic resonance in children and adults with congenital heart disease: SCMR expert consensus group on congenital heart disease. J Cardiovasc Magn Reson. 2013 Jun;15(1):51.

9 Lotz J, Meier C, Leppert A, Galanski M. Cardiovascular flow measurement with phase-contrast MR imaging: basic facts and implementation. Radiographics. 2002 May-Jun;22(3):651-71.

10 Muthurangu V, Taylor A, Andriantsimiavona R, Hegde S, Miquel ME, Tulloh R, et al. Novel method of quantifying pulmonary vascular resistance by use of simultaneous invasive pressure monitoring and phase-contrast magnetic resonance flow. Circulation. 2004 Aug;110(7):826-34.
11 Wright GA, Hu BS, Macovski A. 1991 I.I. Rabi Award. Estimating oxygen saturation of blood in vivo with MR imaging at $1.5 \mathrm{~T}$. J Magn Reson Imaging. 1991 May-Jun;1(3): 275-83.

12 Portnoy S, Milligan N, Seed M, Sled JG, Macgowan CK. Human umbilical cord blood relaxation times and susceptibility at 3 T. Magn Reson Med. 2018 Jun;79(6):3194-206.

13 Roy CW, Marini D, Lloyd DF, Mawad W, Yoo SJ, Schrauben EM, et al. Preliminary Experience Using Motion Compensated CINE Magnetic Resonance Imaging to Visualise Fetal Congenital Heart Disease. Circ Cardiovasc Imaging. 2018 Dec;11(12):e007745.

14 Seed M, van Amerom JF, Yoo SJ, Al Nafisi B, Grosse-Wortmann L, Jaeggi E, et al. Feasibility of quantification of the distribution of blood flow in the normal human fetal circulationusing CMR: a cross-sectional study. J Cardiovasc Magn Reson. 2012 Nov;26;14: 79. 
15 Seed M. Advanced Fetal Cardiac MRI. In: Kline-Fath B, Bulas D, Bahado-Singh R, editors. Diagnostic imaging of fetal anomalies: ultrasound and MRI. Groningen: Wolters Kluwer; 2015. p. 228-34.

16 Prsa M, Sun L, van Amerom J, Yoo SJ, GrosseWortmann L, Jaeggi E, et al. Reference ranges of blood flow in the major vessels of the normal human fetal circulation at term by phasecontrast magnetic resonance imaging. Circ Cardiovasc Imaging. 2014 Jul;7(4):663-70.

17 Roy CW, Seed M, van Amerom JF, Al Nafisi B, Grosse-Wortmann L, Yoo SJ, et al. Dynamic imaging of the fetal heart using metric optimized gating. Magn Reson Med. 2013 Dec; 70(6):1598-607.

18 Roy CW, Seed M, Kingdom JC, Macgowan CK. Motion compensated cine CMR of the fetal heart using radial undersampling and compressed sensing. J Cardiovasc Magn Reson. 2017;19:29.

19 Harris AD, Saleh MG, Edden RA. Edited $1 \mathrm{H}$ magnetic resonance spectroscopy in vivo: Methods and metabolites. Magn Reson Med. 2017 Apr;77(4):1377-1389.

20 Kording F, Yamamura J, de Sousa MT, Ruprecht C, Hedström E, Aletras AH, et al. Dynamic fetal cardiovascular magnetic resonance imaging using Doppler ultrasound gating. J Cardiovasc Magn Reson. 2018 Mar;20(1):17.

21 Bidhult S, Töger J, Heiberg E, Carlsson M, Arheden $\mathrm{H}$, Aletras $\mathrm{AH}$, et al. Independent validation of metric optimized gating for fetal cardiovascular phase-contrast flow imaging. Magn Reson Med. 2019 Jan;81(1):495-503.

22 Bhat M, Haris K, Bidhult S, Liuba P, Aletras $\mathrm{AH}$, Hedström E. Fetal iGRASP cine CMR assisting in prenatal diagnosis of complicated cardiac malformation with impact on delivery planning. Clin Physiol Funct Imaging. 2019 Jul;39(4):231-5.

23 Lloyd DF, Pushparajah K, Simpson JM, van Amerom JF, van Poppel MP, Schulz A, et al. Three-dimensional visualisation of the fetal heart using prenatal MRI with motion-corrected slice-volume registration: a prospective, single-centre cohort study. Lancet. 2019 Apr;393(10181):1619-27.

24 Ray JG, Vermeulen MJ, Bharatha A, Montanera WJ, Park AL. Association Between MRI Exposure During Pregnancy and Fetal and Childhood Outcomes. JAMA. 2016 Sep; 316(9):952-61.

25 Kanal E, Barkovich AJ, Bell C, Borgstede JP, Bradley WG Jr, Froelich JW, et al.; Expert Panel on MR Safety. ACR guidance document on MR safe practices: 2013. J Magn Reson Imaging. 2013 Mar;37(3):501-30.

26 Yagel S, Silverman N, Gembruch U, editors. Fetal Cardiology. Boca Raton: Taylor \& Francis Group; 2019.

27 Schrauben EM, Saini BS, Darby JR, Soo JY, Lock MC, Stirrat E, et al. Fetal hemodynamics and cardiac streaming assessed by $4 \mathrm{D}$ flow cardiovascular magnetic resonance in fetal sheep. J Cardiovasc Magn Reson. 2019 Jan; 21(1):8.
28 Limperopoulos C, Tworetzky W, McElhinney DB, Newburger JW, Brown DW, Robertson RL Jr, et al. Brain volume and metabolism in fetuses with congenital heart disease: evaluation with quantitative magnetic resonance imaging and spectroscopy. Circulation. 2010 Jan;121(1):26-33.

29 Sun L, Macgowan CK, Sled JG, Yoo SJ, Manlhiot C, Porayette $\mathrm{P}$, et al. Reduced fetal cerebral oxygen consumption is associated with smaller brain size in fetuses with congenital heart disease. Circulation. 2015 Apr;131(15):1313-23.

30 Zhu MY, Stochitoiu IA, Jaeggi E, Yoo SJ, Grosse-Wortmann L, Macgowan C, et al. Combined ventricular output and oxygen delivery are reduced while oxygen extraction fraction is increased in fetuses with Ebstein's Anomaly by MRI. J Cardiovasc Magn Reson. 2016;18(S1 Suppl 1):O71.

31 Torigoe T, Mawad W, Seed M, Ryan G, Marini D, Golding F, et al. Treatment of fetal circular shunt with non-steroidal anti-inflammatory drugs. Ultrasound Obstet Gynecol. 2019 Jun;53(6):841-846.

32 Jones HN, Olbrych SK, Smith KL, Cnota JF, Habli M, Ramos-Gonzales O, et al. Hypoplastic left heart syndrome is associated with structural and vascular placental abnormalities and leptin dysregulation. Placenta. 2015 Oct;36(10):1078-86.

33 Goff DA, McKay EM, Davey BR, Thacker D, Khalek N, Miesnik SR, et al. Abstract 11260: placental abnormalities in fetal congenital heart disease. Circulation. 2011;124:A11260.

34 Albalawi A, Brancusi F, Askin F, Ehsanipoor $\mathrm{R}$, Wang J, Burd I, et al. Placental characteristics of fetuses with congenital heart disease. J Ultrasound Med. 2017 May;36(5):965-72.

35 Lauridsen MH, Uldbjerg N, Henriksen TB, Petersen OB, Stausbøl-Grøn B, Matthiesen NB, et al. Cerebral Oxygenation Measurements by Magnetic Resonance Imaging in Fetuses With and Without Heart Defects. Circ Cardiovasc Imaging. 2017 Nov; 10(11):e006459.

36 Homsy J, Zaidi S, Shen Y, Ware JS, Samocha KE, Karczewski KJ, et al. De novo mutations in congenital heart disease with neurodevelopmental and other congenital anomalies. Science. 2015 Dec;350(6265):1262-6.

37 Porayette P, Madathil S, Sun L, Jaeggi E, Grosse-Wortmann L, Yoo SJ, et al. MRI reveals hemodynamic changes with acute maternal hyperoxygenation in human fetuses with and without congenital heart disease. Prenat Diagn. 2016 Mar;36(3):274-81.

38 Edwards LA, Lara DA, Sanz Cortes M, Hunter JV, Andreas S, Nguyen MJ, et al. Chronic Maternal Hyperoxygenation and Effect on Cerebral and Placental Vasoregulation and Neurodevelopment in Fetuses with Left Heart Hypoplasia. Fetal Diagn Ther. 2019;46(1):4557.

39 Zhu MY, Milligan N, Keating S, Windrim R, Keunen J, Thakur V, et al. The hemodynamics of late-onset intrauterine growth restriction by MRI. Am J Obstet Gynecol. 2016 Mar; 214(3):367.e1-17.
40 Siauve N, Chalouhi GE, Deloison B, Alison M, Clement $\mathrm{O}$, Ville $\mathrm{Y}$, et al. Functional imaging of the human placenta with magnetic resonance. Am J Obstet Gynecol. 2015 Oct;213(4 Suppl):S103-14.

41 Sørensen A, Peters D, Simonsen C, Pedersen M, Stausbøl-Grøn B, Christiansen OB, et al. Changes in human fetal oxygenation during maternal hyperoxia as estimated by BOLD MRI. Prenat Diagn. 2013 Feb;33(2):141-5.

42 McKelvey SS, Kay HH. Magnetic resonance spectroscopy of the placenta. Placenta. 2007 May-Jun;28(5-6):369-77.

43 Seed M, Bradley T, Bourgeois J, Jaeggi E, Yoo SJ. Antenatal MR imaging of pulmonary lymphangiectasia secondary to hypoplastic left heart syndrome. Pediatr Radiol. 2009 Jul; 39(7):747-9.

44 Borik S, Macgowan CK, Seed M. Maternal hyperoxygenation and foetal cardiac MRI in the assessment of the borderline left ventricle. Cardiol Young. 2015 Aug;25(6):1214-7.

45 Porayette P, van Amerom JF, Yoo SJ, Jaeggi E, Macgowan CK, Seed M. MRI shows limited mixing between systemic and pulmonary circulations in foetal transposition of the great arteries: a potential cause of in utero pulmonary vascular disease. Cardiol Young. 2015 Apr;25(4):737-44.

46 Al Nafisi B, van Amerom JF, Forsey J, Jaeggi E, Grosse-Wortmann L, Yoo SJ, et al. Fetal circulation in left-sided congenital heart disease measured by cardiovascular magnetic resonance: a case-control study. J Cardiovasc Magn Reson. 2013 Jul;15(1):65.

47 Lloyd DF, van Amerom JF, Pushparajah K, Simpson JM, Zidere V, Miller O, et al. An exploration of the potential utility of fetal cardiovascular MRI as an adjunct to fetal echocardiography. Prenat Diagn. 2016 Oct;36(10): 916-25.

48 Jørgensen DS, Vejlstrup N, Rode L, Ekelund CK, Macgowan CK, Jensen LN, et al. Magnetic Resonance Imaging: A New Tool to Optimize the Prediction of Fetal Anemia? Fetal Diagn Ther. 2019;46(4):257-65.

49 Duan A, Keunen J, Portnoy, Zhu M, Anastasiadis C, Ryan G, et al. Preliminary investigation of the utility of MRI for measuring the hematocrit in fetal anemia. Ultrasound $\mathrm{Ob}$ stet Gynecol. 2016 Sep;48(S1):237.

50 Duan AQ, Lock MC, Perumal SR, Darby JR, Soo JY, Selvanayagam JB, et al. Feasibility of detecting myocardial infarction in the sheep fetus using late gadolinium enhancement CMR imaging. J Cardiovasc Magn Reson. 2017 Sep;19(1):69.

51 Duan AQ, Darby JR, Soo JY, Lock MC, Zhu MY, Flynn LV, et al. Feasibility of phase-contrast cine magnetic resonance imaging for measuring blood flow in the sheep fetus. Am J Physiol Regul Integr Comp Physiol. 2019 Dec;317(6):R780-R792. 